\title{
Robot-Assisted Thoracic Surgery Thymectomy
}

\author{
Samina Park, M.D. \\ Department of Thoracic and Cardiovascular Surgery, Seoul National University Hospital, Seoul National University College of Medicine, Seoul, Korea
}

\section{ARTICLE INFO}

Received June 7, 2021

Accepted July 2, 2021

Corresponding author

Samina Park

Tel $82-2-2072-2345$

Fax 82-2-764-3664

E-mail sam841203@snu.ac.kr

ORCID

https://orcid.org/0000-0001-9625-2672
Robotic thymectomy has been adopted recently and has been shown to be safe and feasible in treating thymic tumors and myasthenia gravis. The surgical indications of robotic technology are expanding, with advantages including an excellent surgical view and sophisticated manipulation. Herein, we describe technical aspects, considerations, and outcomes of robotic thymectomy.

Keywords: Robotic surgical procedures, Thymectomy, Minimally invasive surgery, Thymic neoplasms, Myasthenia gravis

\section{Introduction}

The minimally invasive approach has emerged as an alternative to median sternotomy for thymectomy. Minimally invasive thymectomy has been widely performed using thoracoscopy [1-4]. Since the first robotic thymectomy was reported in 2003 by Ashton et al. [5], it has been widely accepted as an effective surgical option for thymic epithelial tumors (TET) and myasthenia gravis (MG) [6-9]. From the standpoint of surgical outcomes, robotic thymectomy has shown excellent safety and perioperative results, with comparable outcomes to open sternotomy in terms of oncologic survival and neurologic improvement [1,2,9-11]. Robotic thymectomy is commonly performed via the lateral or subxiphoid approach [12-15]. Technical advances in robotic surgery have expanded the indications for robotic thymectomy. Furthermore, single-port and single-site platforms are also applied for treating anterior mediastinal masses [16-18]. In this article, technical details, considerations, and outcomes of robotic thymectomy are described.

\section{Surgical anatomy}

The thymus is a lymphoid organ located in the anterior part of the superior mediastinum, extending into the anterior mediastinum. It is composed of 2 lobes covered with loose connective tissue. The thymus lies behind the sternum and strap muscles, and anterior to the pericardium, the innominate vein, superior vena cava, ascending aorta, aortic arch, and arch vessels. It is connected to the lower poles of the thyroid gland by the thyrothymic ligament. Laterally, the phrenic nerves and mediastinal pleura run along the thymus bilaterally. The thymic arteries mainly comprise (1) the lateral thymic artery from the internal mammary artery and the pericardiacophrenic artery, (2) the superior thymic artery from the inferior thyroid artery, and (3) the posterior thymic artery from the aorta or innominate artery. The thymic veins comprise (1) the posterior thymic vein to the innominate vein, and (2) small tributaries to the inferior thyroid vein, internal mammary vein, and superior vena cava. The lymphatic vessels drain to the parasternal, brachiocephalic, and tracheobronchial lymph nodes.

\section{Indications}

Total thymectomy is primarily indicated for TET and MG [19,20]. Based on institutional experience, en bloc resection of the invaded organs, including the pericardium, lung, phrenic nerve, and innominate vein, is safely performed by robotic thymectomy. However, advanced thymic tumors invading the chest wall or great vessels and large tumors (over $8 \mathrm{~cm}$ ) are considered contraindications of robotic thymectomy $[15,21]$. 


\section{Robotic thymectomy via the subxiphoid approach}

Using robotic technology in addition to the subxiphoid approach may maximize the advantages of the subxiphoid approach by enabling meticulous dissection and safe manipulation around the upper mediastinum, even in advanced tumors. Since robotic thymectomy via the subxiphoid approach (RTX) was reported by Suda et al. [22], several advantages of the subxiphoid approach in thymectomy have been suggested, including (1) an excellent view of the bilateral upper poles of the thymus and bilateral phrenic nerves, (2) minimal pain from not spreading the intercostal space, and (3) excellent cosmesis $[4,14,23,24]$. Herein, the details of RTX are described.

\section{Preparation}

A double-lumen endotracheal tube is routinely used during thymectomy, but single-lung ventilation is limited to selective application in cases involving concomitant lung resection and/or identification of the dislocated phrenic nerve due to a large tumor. Central catheterization is not used. The patient is positioned supine with a $10-\mathrm{cm}$ depth of the pad under the back and both arms abducted to prevent interference with the movement of the robotic arms (Fig. 1). The table is not tilted and the patient is not placed in the reverse Trendelenburg position. No additional instrument for sternal elevation is used. Fig. 2 shows a typical port placement for 3-arm RTX. A 3- to 4 -cm vertical incision is made at the lower half of the xiphoid process. After dividing the linea alba, the xiphoid process is partially resected to widen the subxiphoid space and reduce colli-

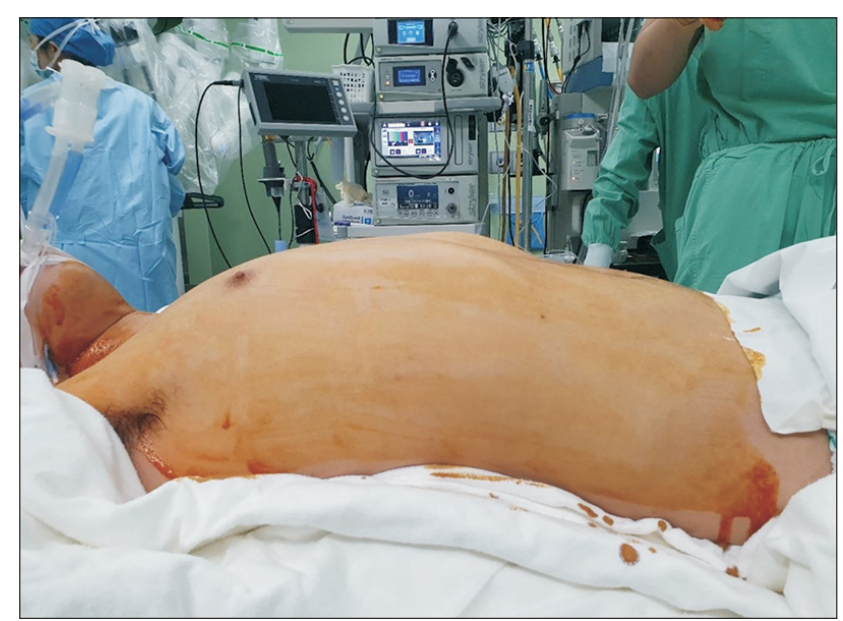

Fig. 1. The patient is positioned supine with a $10-\mathrm{cm}$ depth of the pad under the back and both arms abducted. sions between the camera arm and the sternum. The bilateral mediastinal pleura is opened using electrocautery under thoracoscopic guidance while an assistant holds the sternum upward to ensure sufficient space between the sternum and pericardium (Fig. 2). Then, a 4-channel glove port inserted through the subxiphoid window allows $\mathrm{CO}_{2}$ insufflation, which makes a wide substernal space and collapses the lungs through the pleural opening. Two additional robotic ports are safely inserted at the bilateral fifth intercostal space along the mid-point between the midclavicular line and the anterior axillary line (Fig. 3). Most thymectomies can be completed using the 3 -arm technique (Maryland bipolar forceps for the first robotic arm, $30^{\circ}$ camera for the second arm, and Harmonic ACE curved shears or Monopolar curved scissors [Hot Shears] for the third arm); however, advanced cases require an additional

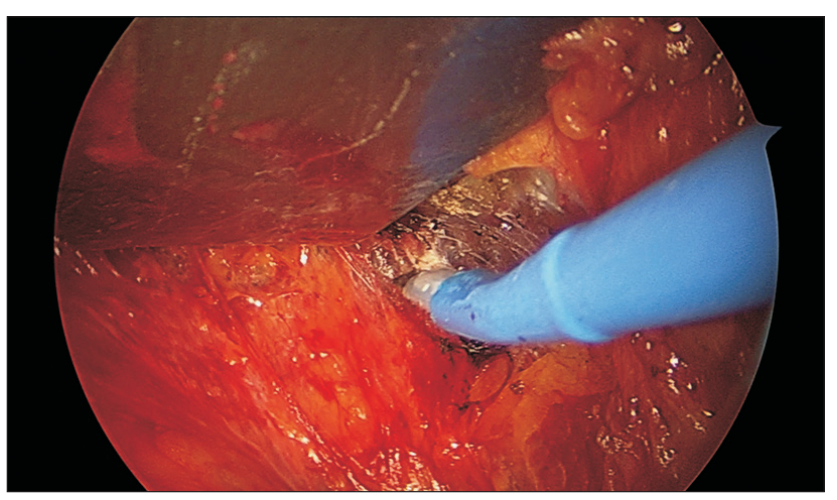

Fig. 2. The bilateral mediastinal pleura is opened using electrocautery under thoracoscopic guidance.

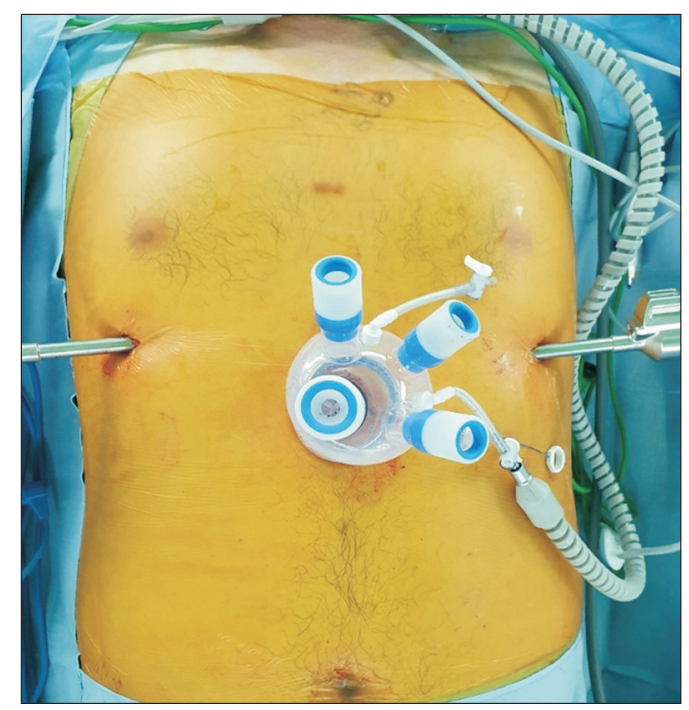

Fig. 3. Typical port placement for 3-arm robotic thymectomy via the subxiphoid approach. 
holding arm (Force bipolar for the fourth arm), which is placed in the third or fourth intercostal space at the anterior axillary line. After docking the robot, the patient cart is placed above the patient's head. The da Vinci Si System can be used for the subxiphoid approach in most cases, but it cannot be utilized effectively in some advanced cases. Moreover, the 12-mm camera of the Si System induces frequent collisions between the sternum and the camera and reduces the flexibility to approach the patient's bed from multiple directions. Therefore, to benefit from the advantages of the subxiphoid approach, the da Vinci Xi System is more suitable than the Si System.

\section{Retrosternal dissection and pleural cavity exploration}

The thymus is separated with Harmonic ACE curved shears from the sternum via $30^{\circ}$ upward vision. The strap muscles and bilateral internal mammary vessels are useful anatomical boundaries for total thymectomy. Then, the angle of the camera turns to $30^{\circ}$ down and the bilateral pleural cavity should be explored to identify unexpected pleural seeding nodules, tumor invasion to adjacent organs, and the location of the bilateral phrenic nerves.

\section{Dissection of the bilateral upper poles and innominate vein}

Upper pole dissection is relatively difficult in the lateral approach. However, in the subxiphoid approach, the trachea, the innominate artery, and the sternal notch are easily accessible without collision of instruments or limited vision. Complete dissection of the bilateral upper poles from the thyroid gland is possible in most cases (Fig. 4). The thymic vasculature is relatively inconsistent. However,

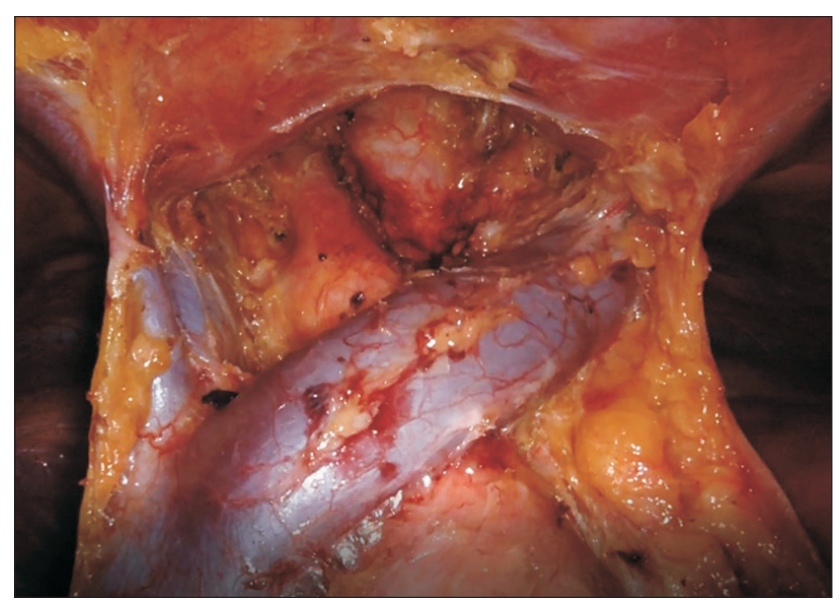

Fig. 4. After dissecting the bilateral upper poles and innominate vein, the upper mediastinum is clearly exposed. most thymic vessels are small enough to be divided with a robotic energy device. Bipolar electrocautery or ligation clips can also be applied according to the surgeon's preference. In addition, the Harmonic ACE curved shears for the third arm is not an angulated device; therefore, dissection from the left lobe to the right lobe is helpful for safely dividing venous tributaries draining to the innominate vein.

\section{Dissection of bilateral lower poles}

Separation of the lower poles of the thymus from the pericardium can be easily performed with a robotic energy device. For small tumors without invasion of the adjacent organs, the entire procedure is conducted with a robotic energy device. In cases of tumors abutting or invading the phrenic nerves or the pericardium, robotic monopolar curved scissors are suitable for a precise dissection. In addition, the robotic camera can be inserted laterally in the contralateral direction through the subxiphoid window to improve the exposure of the diaphragmatic fat.

\section{Specimen retrieval}

While $\mathrm{CO}_{2}$ gas is insufflated, the endo bag through 1 channel of a glove port is placed in the pleural cavity under the guidance of the robotic camera inserted in the port for the first robotic arm. The resected thymus is brought into the endo bag using the third robotic arm to avoid injuring the tumor or adjacent organs. Then, the specimen is retrieved via the subxiphoid window. Additional extension of the wound is rarely required because the substernal space is quite flexible.

\section{Advantages of RTX}

RTX enables extensive resection and meticulous dissection in stage II-III TET and tumors located in the upper mediastinum. Therefore, the following potential advantages of RTX over other minimally invasive approaches are suggested.

\section{Easy to learn}

The surgical view is similar to traditional sternotomy. In addition, the instruments used during the procedure are simple enough to be familiar and the assistant's help is rarely required. Furthermore, if open sternotomy is required, the subxiphoid window can immediately be extended to median sternotomy. Therefore, novice surgeons who would like to start performing robotic thoracic surgery are recommended to start with RTX for small tumors as their first robotic procedure. 


\section{Feasible for advanced or large tumors}

The excellent exposure of the superior mediastinum from the subxiphoid approach, in addition to the advantages of the robotic technique, allows surgeons to perform a fine dissection for complete resection and complex procedures such as reconstruction of the pericardium, vessels, and nerves, which were previously considered to require open sternotomy [25,26]. (1) Pericardium: Pericardial resection and reconstruction can easily be completed with robotic instruments. Pericardial defects are reconstructed with a Gore-Tex membrane using a robotic needle driver. The preferred suture materials are silk and polypropylene. (2) Lung: En bloc wedge resection of the invaded lung is carried with robotic staplers (Fig. 5). If concomitant lobectomy during RTX is needed for a tumor invading the pulmonary hilum, the modified subxiphoid approach can be applied [27]. (3) Innominate vein: The innominate vein can be transected or partially resected with robotic staplers (Fig. 6). If the innominate vein should be reconstructed, vascular clamps are useful to control the proximal and distal part of the vessel during the reconstruction using a vascular prosthesis [25]. (4) Phrenic nerve: Firefly vision is useful to find pericardiacophrenic vessels if the phrenic nerve is not clearly defined in obese patients or tumors overriding the nerve [28]. Neurorrhaphy is also feasible using robotic technology for a partially resected phrenic nerve.

\section{Easy to modify}

Some modifications can be made by changing the location of the ports and the direction of the approach. According to the location or size of tumors, inserting the robotic camera into the ipsilateral thoracic cavity through

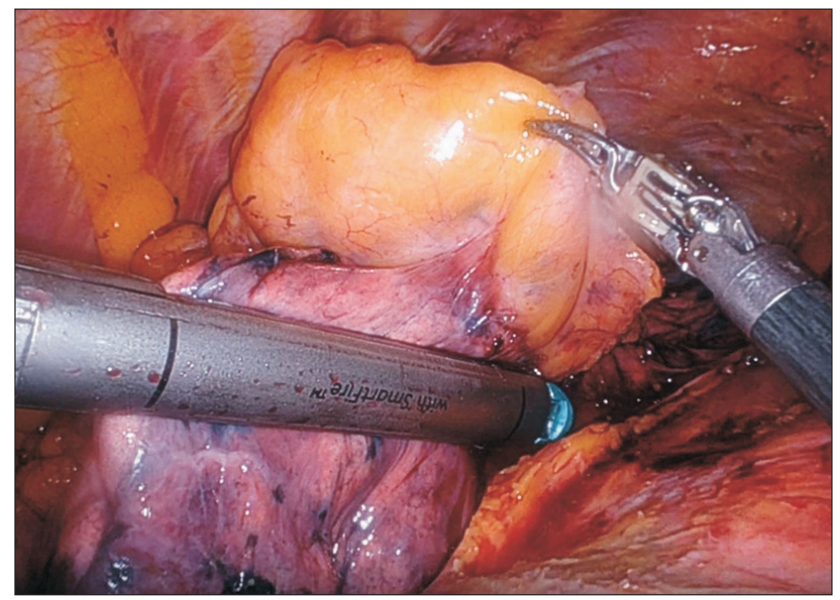

Fig. 5. A robotic stapler is used for en bloc wedge resection of the right upper lobe during robotic thymectomy. the fifth intercostal space is an alternative method (modified subxiphoid approach) [27]. Furthermore, the camera inserted through the intercostal port provides an excellent surgical view around the tumor and the pulmonary hilum. It also can be modified to uni-portal surgery using a single-site platform for limited cases [17].

\section{Feasible for lymph node dissection}

Anterior (perithymic) lymph nodes (N1) can be removed during total thymectomy. However, deep regional lymph nodes (N2) cannot be dissected using conventional minimally invasive approaches due to the limited surgical view. However, the right and left paratracheal areas can be safely accessed by RTX. The subcarinal area can be approached through the modified subxiphoid approach.

\section{Preserves the intercostal space and bundles}

An important aspect of the subxiphoid approach is its ability to spare the intercostal muscles and bundles, which is associated with reducing intercostal neuralgia. Unlike the lateral approach, large tumors can be retrieved via a subxiphoid window without additional extension of the wound in most cases.

\section{Robotic thymectomy via the lateral approach}

Robotic thymectomy via the lateral approach has been more commonly performed than RTX. The lateral approach is performed via the right side, left side, or bilaterally, according to the extent and location of the mass or the surgeon's preference. However, the bilateral approach is generally preferred for patients with MG. The patient is placed in the supine position with elevation of the side to

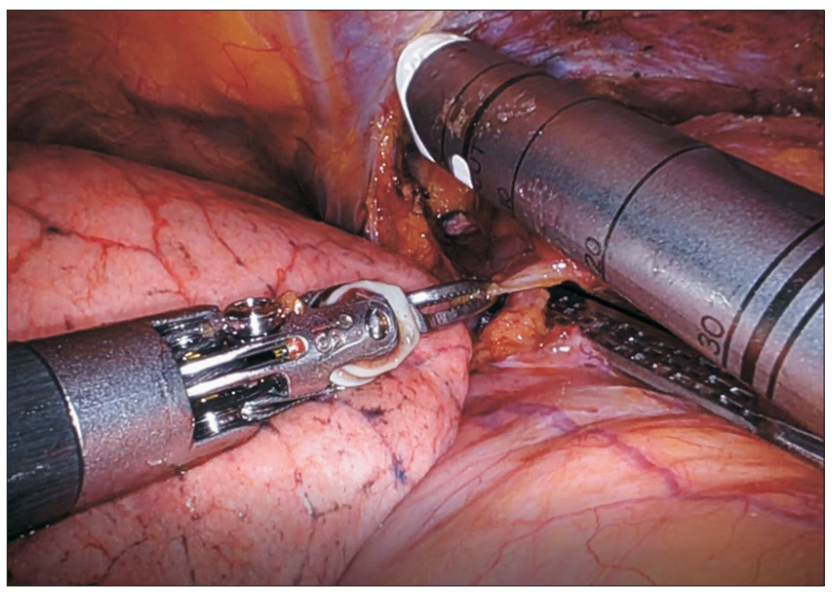

Fig. 6. The proximal innominate vein is divided with a robotic stapler. 
be approached. The adduction and lower positioning of the ipsilateral arm enable the robotic arm and trocar near the axilla to move more freely without injuring the patient by pressing. The 3-arm technique is commonly used. The camera port is inserted at the fourth or fifth intercostal space along the mid-axillary line. Then, $\mathrm{CO}_{2}$ gas is insufflated under single-lung ventilation. $\mathrm{CO}_{2}$ insufflation is mandatory during the entire procedure and provides a wide substernal space to manipulate the dissected thymus under clear vision. The pressure is initially $5 \mathrm{~mm} \mathrm{Hg}$ and is increased to $12 \mathrm{~mm} \mathrm{Hg}$ gradually under stable vital signs, if required. The instruments used are Maryland bipolar forceps for the first robotic arm at the fourth or fifth intercostal space along the mid-axillary line, and a Harmonic ACE curved shears or monopolar curved scissors for the third arm at the fifth or sixth intercostal space along the anterior axillary line. An assistant for the suction device can be additionally used between the camera port and third arm port, if necessary. After docking of the robotic arms, thymectomy is performed in the same manner through conventional thoracoscopic thymectomy via the lateral approach. Therefore, skillful thoracoscopic surgeons may be familiar with the adoption of robotic techniques in thymectomy via the lateral approach.

\section{Postoperative management}

A single Jackson-Pratt drain is placed across the substernal area and is removed on the operative day. Patients are usually discharged on the day after surgery. When concomitant lung resection is performed, a chest tube is inserted. Patients who are expected to have respiratory difficulty due to MG or phrenic nerve resection should only be managed in the intensive care unit.

\section{Outcomes of robotic thymectomy}

The safety and feasibility of robotic thymectomy for TET have been reported, with a complication rate less than 5\% $[14,15,21,22,24]$. In addition, oncologic outcomes were satisfactory (5-year disease-specific survival of $100 \%$ in thymoma and $95 \%$ in thymic carcinoma) [21]. Neurological benefits and decreased use of steroids in MG were observed after robotic thymectomy (complete remission rates of 39\% in robotic thymectomy and $20 \%$ in nonrobotic thymectomy) $[29,30]$. No studies have yet compared the subxiphoid and lateral approaches in robotic thymectomy. However, it is reasonable to predict that RTX would be more suitable for advanced and highly located tumors due to the innate characteristics of RTX. Two meta-analyses of thoracoscopic thymectomy revealed that the subxiphoid approach was associated with less blood loss, a lower pain score, and a shorter hospital stay compared to the lateral approach $[31,32]$. Furthermore, there were no differences in conversion to open sternotomy and oncologic outcomes between the 2 approaches [31]. Randomized studies comparing open sternotomy, the thoracoscopic approach, and the robotic approach would seem to have difficulty in enrolling patients in practice because the indications for each approach are quite different. Obviously, considering the maximal utilization of robotic techniques in thymectomy, more extensive thymectomy would be available than with thoracoscopic thymectomy.

\section{Conclusion}

Median sternotomy was the gold standard in thymectomy for decades. However, minimally invasive thymectomy has gained popularity because it has demonstrated comparable outcomes to those of open sternotomy. More recently, the robotic approach has become widely adopted in thymectomy. In addition, the subxiphoid approach, which provides an excellent surgical view for highly located tumors and the bilateral phrenic nerves, has been introduced. Thymectomy should be conducted following oncologic principles, regardless of the surgical technique. Furthermore, cosmetic benefits never take precedence over safe and complete surgery. Therefore, surgeons should be familiar with the features of various surgical techniques and utilize the advantages of each approach during thymectomy. The superiority of robotic thymectomy over other minimally invasive approaches or the lateral approach should be investigated in the future.

\section{Conflict of interest}

No potential conflict of interest relevant to this article was reported.

\section{ORCID}

Samina Park: https://orcid.org/0000-0001-9625-2672

\section{References}

1. Jurado J, Javidfar J, Newmark A, et al. Minimally invasive thymectomy and open thymectomy: outcome analysis of 263 patients. Ann Thorac Surg 2012;94:974-81. 
2. Cheng YJ, Kao EL, Chou SH. Videothoracoscopic resection of stage II thymoma: prospective comparison of the results between thoracoscopy and open methods. Chest 2005;128:3010-2.

3. Kido T, Hazama K, Inoue Y, Tanaka Y, Takao T. Resection of anterior mediastinal masses through an infrasternal approach. Ann Thorac Surg 1999;67:263-5.

4. Suda T, Sugimura H, Tochii D, Kihara M, Hattori Y. Single-port thymectomy through an infrasternal approach. Ann Thorac Surg 2012;93:334-6.

5. Ashton RC Jr, McGinnis KM, Connery CP, Swistel DG, Ewing DR, DeRose JJ Jr. Totally endoscopic robotic thymectomy for myasthenia gravis. Ann Thorac Surg 2003;75:569-71.

6. Marulli G, Rea F, Melfi F, et al. Robot-aided thoracoscopic thymectomy for early-stage thymoma: a multicenter European study. J Thorac Cardiovasc Surg 2012;144:1125-30.

7. Marulli G, Schiavon M, Perissinotto E, et al. Surgical and neurologic outcomes after robotic thymectomy in 100 consecutive patients with myasthenia gravis. J Thorac Cardiovasc Surg 2013;145:730-5.

8. Ismail M, Swierzy M, Ruckert JC. State of the art of robotic thymectomy. World J Surg 2013;37:2740-6.

9. Ye B, Li W, Ge XX, et al. Surgical treatment of early-stage thymomas: robot-assisted thoracoscopic surgery versus transsternal thymectomy. Surg Endosc 2014;28:122-6.

10. Kang CH, Hwang Y, Lee HJ, Park IK, Kim YT. Robotic thymectomy in anterior mediastinal mass: propensity score matching study with transsternal thymectomy. Ann Thorac Surg 2016;102:895-901.

11. Meyer DM, Herbert MA, Sobhani NC, et al. Comparative clinical outcomes of thymectomy for myasthenia gravis performed by extended transsternal and minimally invasive approaches. Ann Thorac Surg 2009;87:385-90.

12. Chen K, Zhang X, Jin R, et al. Robot-assisted thoracoscopic surgery for mediastinal masses: a single-institution experience. J Thorac Dis 2020;12:105-13

13. Suda T, Kaneda S, Hachimaru A, et al. Thymectomy via a subxiphoid approach: single-port and robot-assisted. J Thorac Dis 2016;8(Suppl 3):S265-71.

14. Zhang H, Chen L, Zheng Y, et al. Robot-assisted thymectomy via subxiphoid approach: technical details and early outcomes. J Thorac Dis 2018;10:1677-82.

15. Kang CH, Na KJ, Song JW, et al. The robotic thymectomy via the subxiphoid approach: technique and early outcomes. Eur J Cardiothorac Surg 2020;58(Suppl_1):i39-43.

16. Ishikawa N, Oda M, Kawachi K, Watanabe G. Robot-assisted single-port surgery for mediastinal tumors. Surg Today 2019;49:96-8.

17. Park SY, Han KN, Hong JI, Kim HK, Kim DJ, Choi YH. Subxiphoid approach for robotic single-site-assisted thymectomy. Eur J Cardiothorac Surg 2020;58(Suppl_1):i34-8.
18. Park SY, Kim HK, Jang DS, Han KN, Kim DJ. Initial experiences with robotic single-site thoracic surgery for mediastinal masses. Ann Thorac Surg 2019;107:242-7.

19. Wolfe GI, Kaminski HJ, Aban IB, et al. Randomized trial of thymectomy in myasthenia gravis. N Engl J Med 2016;375:511-22.

20. Ettinger DS, Riely GJ, Akerley W, et al. Thymomas and thymic carcinomas: clinical practice guidelines in oncology. J Natl Compr Canc Netw 2013;11:562-76.

21. Kang CH, Na KJ, Park S, Park IK, Kim YT. Long-term outcomes of robotic thymectomy in patients with thymic epithelial tumors. Ann Thorac Surg 2020 Oct 29 [Epub]. https://doi.org/10.1016/j.athoracsur.2020.09.018

22. Suda T, Tochii D, Tochii S, Takagi Y. Trans-subxiphoid robotic thymectomy. Interact Cardiovasc Thorac Surg 2015;20:669-71.

23. Chiu CH, Chao YK, Liu YH. Subxiphoid approach for video-assisted thoracoscopic surgery: an update. J Thorac Dis 2018;10(Suppl 14):S1662-5.

24. Hashimoto K, Sakamaki H. The technical aspects of a midline robotic thymectomy. JTCVS Tech 2020;4:368-70.

25. Suda T, Nagano H, Kawai H, Hoshikawa Y. Subxiphoid robot-assisted thymectomy with vascular prosthetic replacement. Semin Thorac Cardiovasc Surg 2020;32:1133-4.

26. Na KJ, Kang CH. Neurorrhaphy of the phrenic nerve injury during robotic thymectomy [Internet]. [place unknown]: ASVIDE; 2020 [cited 2021 May 30]. Available from: https:/www.asvide.com/article/view/33088.

27. Na KJ, Kang CH. Robotic thymectomy for advanced thymic epithelial tumor: indications and technical aspects. J Thorac Dis 2020;12:63-9.

28. Wagner OJ, Louie BE, Vallieres E, Aye RW, Farivar AS. Near-infrared fluorescence imaging can help identify the contralateral phrenic nerve during robotic thymectomy. Ann Thorac Surg 2012;94:622-5.

29. Keijzers M, de Baets M, Hochstenbag M, et al. Robotic thymectomy in patients with myasthenia gravis: neurological and surgical outcomes. Eur J Cardiothorac Surg 2015;48:40-5.

30. Ruckert JC, Swierzy M, Ismail M. Comparison of robotic and nonrobotic thoracoscopic thymectomy: a cohort study. J Thorac Cardiovasc Surg 2011;141:673-7.

31. Li J, Qi G, Liu Y, Zheng X, Zhang X. Meta-analysis of subxiphoid approach versus lateral approach for thoracoscopic thymectomy. J Cardiothorac Surg 2020;15:89.

32. Li M, Xu L, Li L, Dai Q, Xu D. The early perioperative outcomes of subxiphoid approach versus lateral intercostal approach thoracoscopic thymectomy for thymic tumors: a meta-analysis. J Laparoendosc Adv Surg Tech A 2021 Mar 4 [Epub]. https://doi.org/10.1089/ lap.2021.0036. 